\title{
TRAUMA OF THE SPINE AND SPINAL CORD-I
}

\author{
Andrew Swain, John Dove, Harry Baker
}

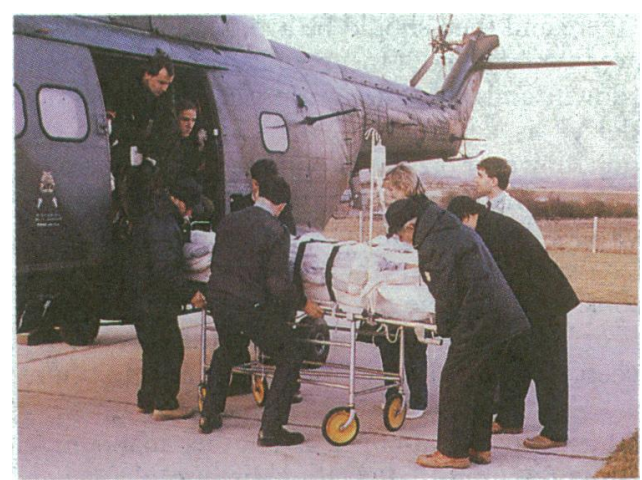

Incidence (percentage) of neurological injury in patients with fractures or dislocations of various parts of the spine

\begin{tabular}{lc}
\hline Part of spine & Incidence \\
\hline Any & 14 \\
Cervical spine & 40 \\
Thoracic spine & 10 \\
Thoracolumbar junction & 35 \\
Lumbar spine & 3
\end{tabular}

A patient with serious multiple injuries is rarely able to provide a coherent history. Injuries that carry a risk of death or severe disability must, therefore, be suspected from the outset so that correct early management can be instituted. Any patient with trauma who is not fully conscious should be assumed to have an injury of the cervical spine until proved otherwise. The thoracolumbar spine must also be managed carefully. The commonest reason for failing to detect an important spinal injury is failure to suspect one, particularly in patients with multiple trauma; but sometimes a serious injury is considered minor.

\section{Management at the scene of the accident}

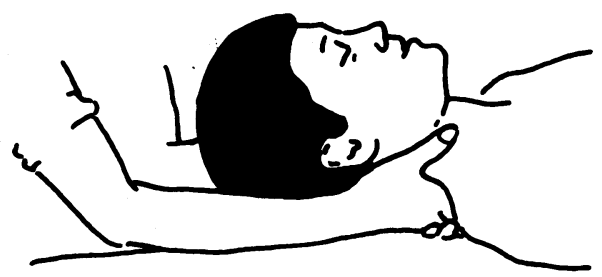

Manual immobilisation of the neck.
The spinal cord is most often damaged in the cervical region, but it is also particularly at risk near the thoracolumbar junction. The thoracic spine is splinted by ribs and sternum, but the spinal canal is narrower in this region relative to the width of the spinal cord so when vertebral displacement occurs it is more likely to damage the cord. Nowadays partial cord injuries are generally more common, and the potential for neurological improvement or deterioration is correspondingly greater.
Spinal trauma may be suspected from a witness's description of an accident. It cannot, however, be excluded without a definitive examination, even in the fully conscious patient. The neck must be aligned in the neutral position without longitudinal compression or distraction. This will improve the airway and reduce spinal deformity, helping to relieve pressure on the spinal cord or arteries. If the patient is a motorcyclist the doctor should support the neck while an assistant carefully eases the helmet off. The neck is then splinted with a rigid collar of appropriate size to grip the chin. Collars alone are inadequate and they need to be supplemented by manual stabilisation or lateral support with, for example, sandbags and forehead tape. Be wary of swelling under the collar, which may develop from a haematoma or surgical emphysema.

In the unconscious patient the airway should be opened by chin or jaw lift and an oropharyngeal airway inserted. The supine position facilitates clinical examination, cardiopulmonary resuscitation (if required), respiratory movements, and control of the neck, but endotracheal intubation is required to prevent aspiration. Alternatively the patient can be turned into the lateral position with the trunk straight but inclined forwards by 20 degrees, allowing secretions to discharge freely from the mouth. The three quarters prone or coma position cannot be recommended as it entails rotation of the cervical spine and splints the diaphragm, causing hypoventilation.
Supine position with airway protection (top); lateral recovery position (bottom). 


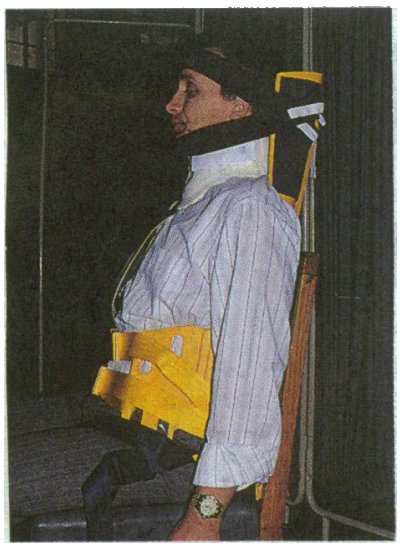

Semi-rigid colla and spine

immobiliser.

\section{Transfer to hospital}

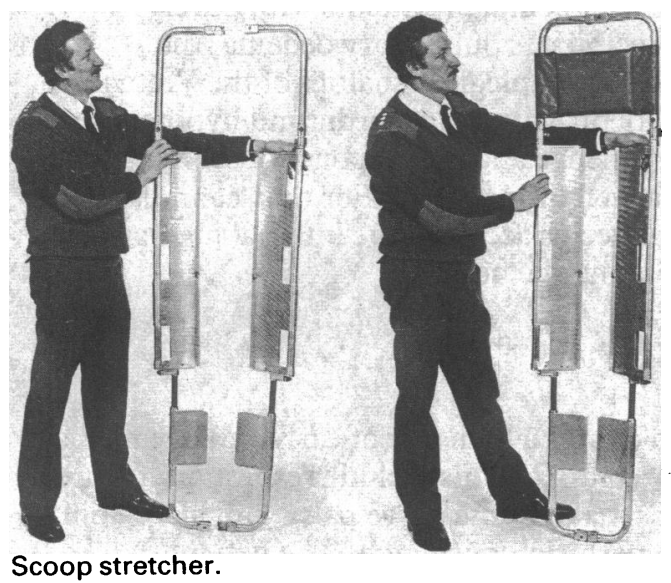

\section{Arrival at hospital: primary survey}

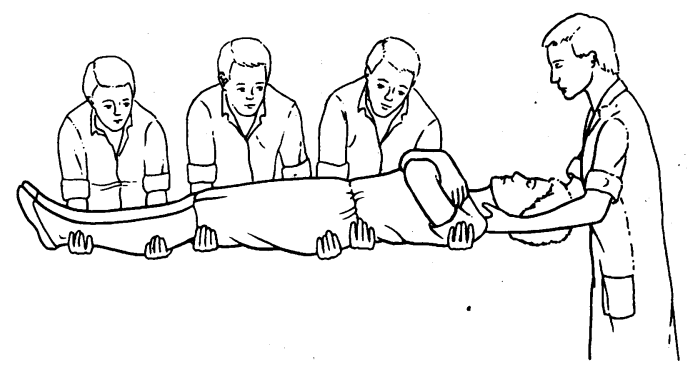

Coordinated spinal lift.

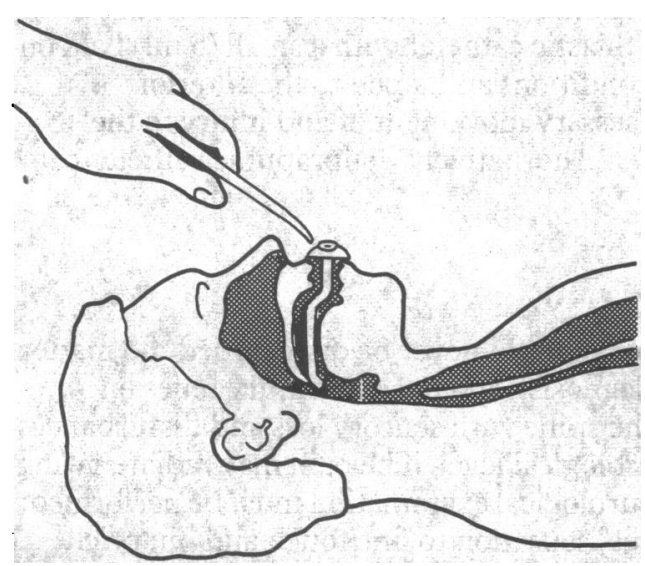

Suction: beware of vagal reflex stimulation.
Thoracolumbar injury must also be assumed and treated by carefully straightening the trunk and correcting rotation. The patient may be log rolled or lifted as necessary (ideally by five assistants), but it is vital that the whole spine is maintained in the neutral position.

The neck and back can be protected simultaneously in patients who are erect or supine by means of a spinal board or one of the more recently developed "spine immobilisers," which are lighter and easier to handle than a board. Doctors should familiarise themselves with the splints that are available locally.

Once the airway is protected and oxygen has been administered and the patient positioned, one or more intravenous infusions are established. If conditions allow, the patient should be examined briefly before transportation. A "scoop" stretcher can be assembled underneath a patient who is lying free and used to transport him or her to the ambulance. Patients in immobilisers must be carried in and not by the splint. In the absence of life threatening injury the patient with spinal trauma should be transported carefully to hospital. Hard objects should be removed from anaesthetic parts of the body.

Safe transfer of the patient from the stretcher to a trauma trolley can be accomplished by four people, the person in charge grasping the base of the neck and supporting the head with his or her wrists. In this way there should be no seesaw movement of the neck if the actions of those lifting the patient become incoordinated. Once the patient is lifted the trauma trolley can be wheeled underneath. Resuscitation is then continued while the spine $\stackrel{N}{\sigma}$ is protected.

\section{Airway (with protection of the cervical spine)}

Patients with multiple trauma are invariably kept supine during resuscitation unless regurgitation occurs and the airway is unprotected. In patients with cervical cord injury pharyngeal stimulation by vigorous suction manipulation of a Guedel airway or intubation may result in unopposed vagal discharge and cardiac arrest. This can be prevented by prior administration of atropine. Many seriously injured patients require intubation, and this is not contraindicated in patients with an unștable cervical injury. The procedure should, however, be performed whenever possible by an experienced anaesthetist and spinal movement minimised by an assistant controlling the head and limiting movement. Alternative methods of intubation that do not require the neck to be moved-for example, blind endotracheal intubation and use of a fibreoptic laryngoscope - should be employed only by those with the necessary experience. Ileus develops after spinal cord injury, and a nasogastric tube is required. 


\section{Treat}

- Bradycardia - pulse rate $<50$ beats/min

- Hypotension-systolic blood pressure $<80 \mathrm{~mm} \mathrm{Hg}$

- Inadequate urinary excretion
Do not rotate the patient's neck during central venous cannulation unless cervical injury has been excluded by radiography

\section{Circulation}

Patients with injury to the cervical cord or high thoracic cord may have reduced sympathetic outflow between the T1 and L 2 segments with associated bradycardia and hypotension. Patients must be connected to a cardiac monitor on admission. Tetraplegic patients with bradycardia should be given atropine if their pulse rate drops below 50 beats $/ \mathrm{min}$. If their systolic blood pressure falls below $80 \mathrm{~mm} \mathrm{Hg}$ inotropic support is necessary. Bradycardia with hypotension is not a classical feature of hypovolaemic shock, and in a traumatised patient it should increase suspicion of spinal cord injury. The extent of bradycardia and hypotension in neurogenic shock depends on the level and extent of neurological injury. Bradycardic shock is also seen in elderly patients and patients taking $\beta$ blockers.

In recent years the importance of maintaining adequate tissue perfusion and oxygenation in patients with spinal cord trauma has been emphasised. Episodes of hypotension or hypoxia often lead to irreversible neurological deterioration. Patients with spinal trauma are likely to have hypovolaemia owing to other injuries. Circulatory volume must be restored, but aggressive fluid replacement is detrimental in patients with purely neurogenic hypotension as it precipitates pulmonary oedema (the commonest cause of early death in tetraplegic casualties of the Vietnam war). Therefore traumatised patients with bradycardia and hypotension should be subjected to a fluid challenge and the response observed and monitored by measuring central venous pressure. For this, cannulation of the subclavian vein is recommended as access to the internal jugular vein is difficult to obtain without rotating the neck.

\section{Secondary survey}

\section{Causes of respiratory insufficiency \\ In tetraplegic patients \\ - Intercostal paralysis \\ - Partial phrenic nerve palsy-immediate - delayed \\ - Impaired ability to expectorate \\ - Ventilation-perfusion mismatch \\ In paraplegic patients \\ - Variable intercostal paralysis according to level of injury \\ - Associated chest injuries - rib fractures, pulmonary contusion, haemopneumothorax}

Method of catheterisation in patients with spinal cord injury.
Any injury to the spinal cord carries a high risk of early and late medical complications. Important early complications include respiratory failure due to intercostal paralysis or partial phrenic nerve palsy, impaired ability to expectorate, and ventilation-perfusion mismatch. The patient's respiratory state may also deteriorate shortly after admission as a result of ascending oedema in the traumatised cervical cord. Care must be taken in giving narcotic analgesics as these will further impair respiration. Cardiac arrest usually results from respiratory failure. Arterial blood gas tensions and vital capacity must be checked.

Abdominal trauma is not easily assessed in tetraplegic patients as the abdominal wall is anaesthetic, flaccid, and areflexic and ileus results from the neurological injury. A useful positive sign is pain at the tip of the shoulder that is aggravated by abdominal palpation. Peritoneal lavage is a particularly useful diagnostic aid in patients with cervical or thoracic cord injury.

Acute retention will develop in paraplegic and tetraplegic patients unless the sacral segments are spared. Measurement of urine output in patients with multiple trauma is important, and the bladder will usually require drainage, particularly if the patient has been drinking. In the absence of urethral trauma a narrow gauge Silastic catheter with a small $(5 \mathrm{ml})$ balloon is passed under strictly aseptic conditions and taped to the anterior abdominal wall to prevent unnecessary movement of and injury to the urethra, which may lead to sepsis. Alternatively a suprapubic catheter can be inserted.

\section{The conscious patient}

Sensory loss or motor symptoms should never be disregarded, no matter how unimportant they seem. Diagnosis of spinal cord injury relies on symptoms and signs of pain in the spine with sensory loss and disturbances in motor function distal to a neurological level. The pain may radiate owing to nerve root irritation. A full neurological examination must be performed, including testing of cranial nerves, sensation to fine touch and pin prick, proprioception, power, tone, and reflexes. 


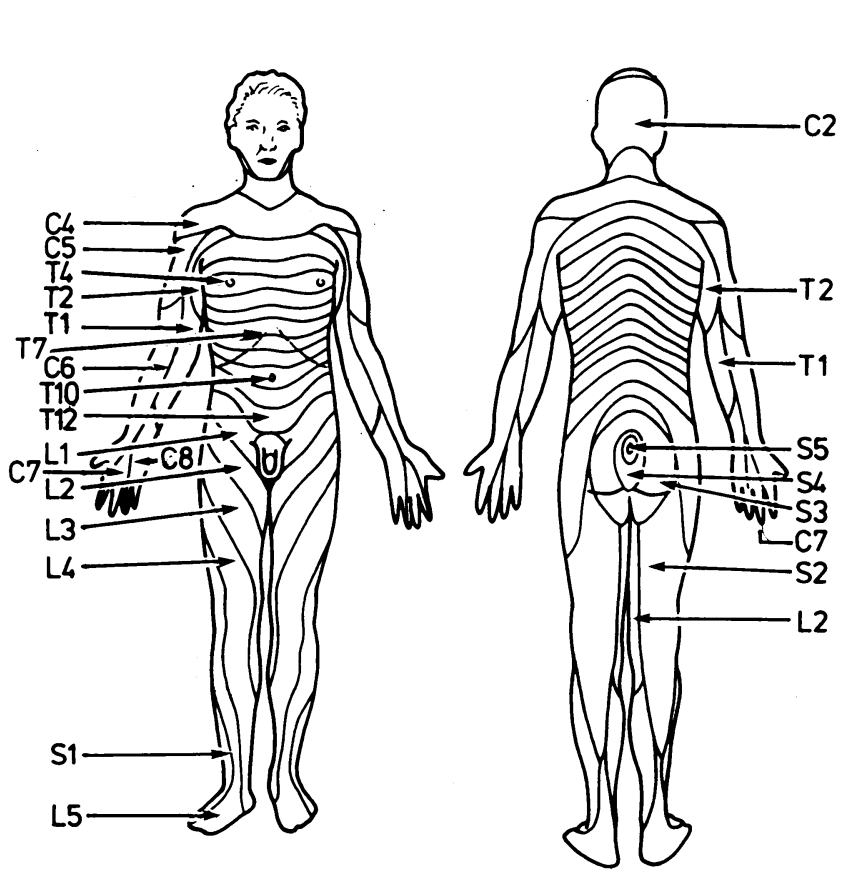

Sensory and motor neurological assessment.

\section{Myotomes}

Muscle group

Diaphragm

Shoulder abductors

Elbow flexors

Supinators/pronators

Wrist extensors

Wrist flexors

Elbow extensors

Finger extensors

Finger flexors

Intrinsic hand muscles T1

Hip flexors
Hip adductors
Knee extensors
Ankle dorsiflexors
Toe extensors
Knee flexors
Ankle plantar flexors
Toe flexors
Anal sphincter

C5

C6

C7

$\mathrm{C7}$

$\mathrm{C} 7$

C8

L5
Reflexes

Nerve supply

$\mathrm{C}(3), 4,(5)$

C5,6 Biceps jerk C5,6

C6 Supinator jerk $\mathrm{C} 6$

Triceps jerk C7

Abdominal reflex T8-12

$L 1,2$

$\mathrm{L} 2,3$

L3,4

$\mathrm{L} 4,5$

L4,5 S1

$\mathrm{S} 1,2$ Ankle jerk S1,2

$\mathrm{S} 1,2$

S2,3,4 Bulbocavernosus reflex S3,4

Anal reflex S5

Plantar reflex

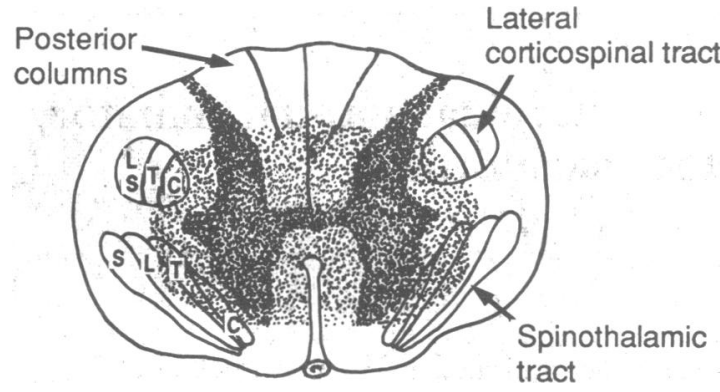

The central cord syndrome.

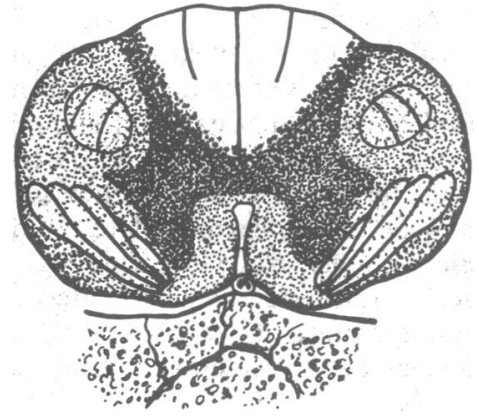

The anterior cord syndrome.

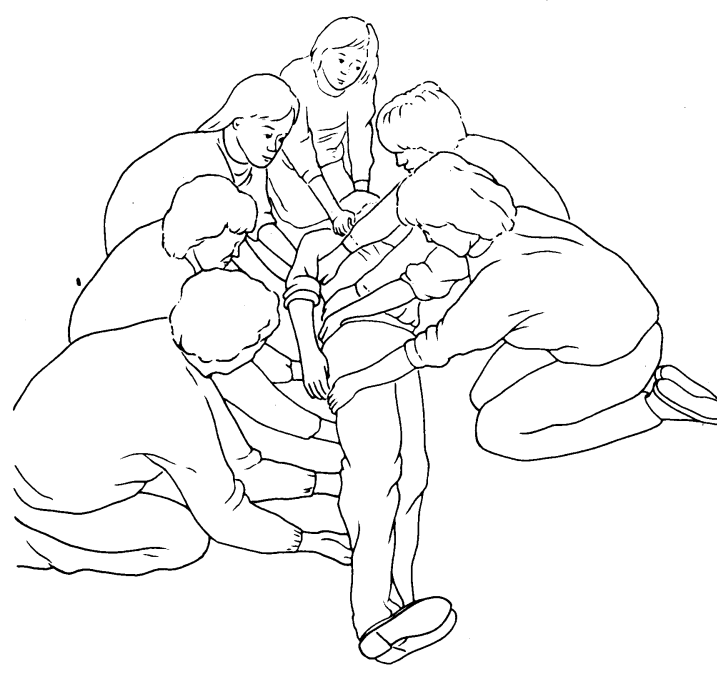

Great care must be taken in managing conscious patients as the neurological symptoms and signs may be dismissed if they do not fit a classical pattern. In patients with a partial cord lesion some neurological function is preserved distal to the level of injury (for example, the sacral segments may be spared): a vascular lesion may be responsible for this. Sometimes the zone of cord injury lies centrally, encroaching on the cervical segments of the long tracts and producing flaccid weakness in the arms (the central cord syndrome). Anterior contusion affects the spinothalamic and corticospinal tracts and is therefore associated with weakness and impaired pain and temperature sensation (the anterior cord syndrome). Posterior cord injury causes loss of sense of vibration and proprioception (the posterior cord syndrome). Trauma may be confined to one side of the cord, producing ipsilateral weakess and impaired contralateral pain and temperature sensation (Brown-Séquard "hemisection"). The central cord syndrome is more common in elderly patients in whom the spinal canal has been narrowed by cervical spondylosis. Patients with this syndrome may not have an associated fracture or dislocation, whereas those with anterior cord injury usually do. Brown-Séquard lesions are more common in patients with penetrating trauma and blunt rotational injury.

\section{The unconscious patient}

There are no truly pathognomic features of spinal cord injury, but important signs are flaccid paralysis, diaphragmatic breathing, priapism, hypotension with bradycardia, and upward movement of the umbilicus on tensing the abdomen - this is due to a T10 lesion (Beavor's sign). Examination of the whole length of the spine must be performed in all unconscious patients with multiple trauma. Failure to do so has resulted in diagnoses being missed, with serious consequences.

The patient is $\log$ rolled on to his or her side, keeping the spine in the neutral position. If performed correctly this is quite safe. Inspection may detect bruising, swelling, or a kyphos; palpation may allow tenderness, an increased interspinous gap, or malalignment of spinous processes (rotational deformity) to be detected. Suspicion of an injury to the upper cervical spine may be aroused by a retropharyngeal haematoma seen through the open mouth; the trachea may be deviated. 


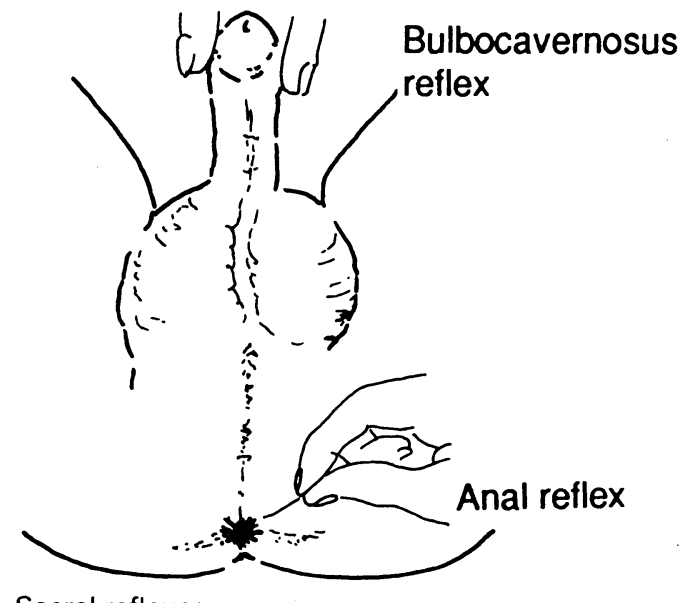

Sacral reflexes.
Neurological examination is mandatory in all unconscious patients, and baseline observations are extremely important in patients with spinal injury, not least for medicolegal reasons. Use of a Glasgow or similar coma chart will allow limb movements and strength to be charted. Some head injury charts do not include this facility. Neurological examination in unconscious patients is usually limited to completing the coma chart, funduscopy, and assessing tone and reflexes, but in patients with suspected cord injury the abdominal, anal, and bulbocavernosus reflexes should be recorded. The sensory response to pain can also be assessed in patients with depressed consciousness. Beware of flaccidity and areflexia in an arm as this may result from brachial plexus injury or spinal cord trauma, or both (particularly in motorcyclists).

The illustrations of the lateral position, transfer to a trauma trolley, and log rolling were prepared by the department of education and medical illustration services, St Bartholomew's Hospital.

Mr Andrew Swain, FRCS, is consultant in accident and emergency medicine, Worcester Royal Infirmary; Mr John Dove, FRCS, is consultant at the Stoke on Trent Spinal Service; Dr Harry Baker, MRCP, is consultant in rehabilitation medicine, Rookwood Hospital, Cardiff.

The ABC of Major Trauma has been edited by Mr David Skinner, FRCS; Mr Peter Driscoll, FRCS; and Mr Richard Earlam, FRCS.

\title{
Lesson of the Week
}

\section{Aplastic anaemia associated with a non-steroidal anti-inflammatory drug: relapse after exposure to another such drug}

\author{
Richard Andrews, Nigel Russell
}

Patients who have had aplastic anaemia while taking a non-steroidal anti-inflammatory drug should avoid subsequent exposure to all such drugs
Aplastic anaemia is one of the most serious of all drug complications and has a high mortality despite advances in treatment. Roughly $30 \%$ of cases are related to environmental factors or drugs. ${ }^{1}$ We report a case of fatal aplastic anaemia related to use of the non-steroidal anti-inflammatory drugs sulindac and fenbufen.

\section{Case report}

A 65 year old woman presented in February 1984 with a four week history of spontaneous bruising and a rash. A full blood count showed a haemoglobin concentration of $75 \mathrm{~g} / 1$, a white cell count of $1.4 \times 10^{9} / 1$ (neutrophils $0.64 \times 10^{\circ} / 1$ ), and a platelet count of $10 \times 10^{9} / 1$. A trephine biopsy of bone showed a severely hypocellular marrow. An acidified serum test (Ham's test) yielded negative results. For the past three years she had been taking propranolol $80 \mathrm{mg}$ twice daily for hypertension and ibuprofen $400 \mathrm{mg}$ thrice daily for osteoarthritis of her knees and spine. Five weeks previously she had been prescribed sulindac $200 \mathrm{mg}$ daily for further analgesia.

Aplastic anaemia was diagnosed and sulindac thought to be the likely causative agent. All analgesics were stopped, a single blood transfusion was given, and oxymetholone $200 \mathrm{mg}$ daily was started. She responded well, and four months later a blood count showed a haemoglobin concentration of $103 \mathrm{~g} / \mathrm{l}$, a white cell count of $5 \cdot 45 \times 10^{9} / 1$ (neutrophils $2 \cdot 8 \times 10^{9} / 1$ ), and a platelet count of $105 \times 10^{9} / 1$. The oxymetholone was stopped because she developed jaundice, but she remained well for three years, not taking any treatment and with blood counts similar to those noted above.

In April 1987 worsening knee pains caused her to consult her general practitioner, who prescribed fenbufen $300 \mathrm{mg}$ during the day and $600 \mathrm{mg}$ at night. Two weeks later she presented with epistaxis, widespread bruising, and a purpuric rash. A full blood count showed recurrent pancytopenia with a haemoglobin concentration of $64 \mathrm{~g} / \mathrm{l}$, a white cell count of $2.6 \times 10^{9} / 1$ (neutrophils $1.6 \times 10^{9} / 1$ ), and a platelet count of $49 \times 10^{9} / 1$. A relapse of her aplastic anaemia was diagnosed on the basis of results of a bone marrow biopsy. Fenbufen was implicated and stopped. She was given a blood transfusion and remained dependent on transfusions for the next six months. Despite further treatment with oxymetholone and antithymocyte globulin her pancytopenia progressively worsened. Her clinical condition deteriorated, and she died of respiratory failure in October 1987. Necropsy showed haemorrhagic pneumonia and aplastic anaemia.

\section{Discussion}

Both sulindac ${ }^{2-5}$ and fenbufen (Committee on Safety of Medicines, personal communication) have been implicated as causative agents in aplastic anaemia. This, however, is the first case to be reported in which a non-steroidal anti-inflammatory drug was implicated in the relapse of aplastic anaemia that had originally been associated with another such drug. We recommend that extreme caution is exercised when any non-steroidal anti-inflammatory drug is given to patients with a history of aplastic anaemia related to such drugs.

\footnotetext{
Gordon-Smith EC. Aplastic anaemia. In: Gordon-Smith EC, ed. Clinics in haematology. Vol 2. No 1. London: Baillière-Tindall, 1989:5-8. Miller JL. Marrow aplasia and sulindac. Ann Intern Med 1980;92:129.

3 Sanz M. Martinez JA, Gomis F, Garcia-Borras JJ. Sulindac induced bone-marrow toxicity. Lancet 1980;ii:802.

4 Bennett L, Schlossman R, Rosenthal J, Balzora JD, Bennet AJ, Rosner F. Aplastic anemia and sulindac. Ann Intern Med 1980;92:874.

5 Anonymous. The international agranulocytosis and aplastic anemia study. Anonymous. The international agranulocytosis and aplastic anemia
Risks of agranulocytosis and aplastic anemia. JAMA 1986;256:1749-57.
}

(Accepted 11 April 1990) 